Pacific Journal of Mathematics

DER HUREWICZ-SATZ 


\section{DER HUREWICZ-SATZ}

\section{FRIEDRICH-WILHELM BAUER}

In [1] F. W. Bauer, Homotopie und Homologie, Mathem. Ann. Bd. 149 S. 105-130 (1963), wurde zu jeder Homologietheorie eine Homotopietheorie gefunden, für die ein Hurewicz-Satz gilt und die mit dieser Eigenschaft universell ist. In dieser Arbeit wird dieser Gedankengang wesentlich verallgemeinert und zu einem beliebigen Funktor $\Phi: \mathfrak{K} \rightarrow \mathfrak{A}(\mathfrak{H}$ ist eine beliebige Kategorie mit hinreichend vielen Nullabbildungen, bezgl. $\mathfrak{A}$ s. 1. Abschnitt) ein eindeutig bestimmter universeller Funktor $\Phi_{\pi}: \Re \rightarrow \mathfrak{U}$ gefunden, der einem Hurewicz-Satz (d. h. Bedingung $H$ ) im 2. Abschnitt) genügt. Die einzige Forderung an $\Phi$ ist eine Art Dimensionsaxiom $\Phi$ 1) im ersten Abschnitt.

Die Konstruktion von $\Phi_{\pi}$ verläuft nach den gleichen Prinzipien wie in [1]. Man hat nur hier bei $\Phi_{\pi}(X)$ i. A. auch dann keine Gruppenstruktur mehr, wenn $\Phi(X)$ noch eine Gruppenstruktur trug. Als unmittelbare Anwendung bekommt man wieder die Hurewiczschen Homotopiegruppen heraus, wenn man von der singulären Homologie ausgeht. Nimmt man einen dualisierten Homotopiefunktor $\pi$ als $\Phi$, so bekommt man fuir $\Phi_{\pi}$ den Cechschen Kohomologiefunktor ${ }^{1}$ heraus. Eine weitere Anwendung ist die Konstruktion eines universellen Homotopiefunktors (d. h. eines solchen, der $H$ ) erfüllt) $\Phi_{w} \mathbf{z u}$ gegebenem $\Phi$, der die folgende Eigenschaft hat:

$W)$ Ist für ein $f \in \Re, \emptyset(f)$ ein Isomorphismus, so auch $\emptyset_{W}(f)$.

Ist $\Phi=H_{n}$, der singuläre Homologiefunktor in irgend einer Dimension, so hat bekanntlich auf einer Kategorie von einfach zusammenhämgenden Räumen der Funktor $\pi_{n}$ diese Eigenschaft. Das ist der Inhalt eines bekannten Satzes von J. H. C. Whitehead. In Satz 4 wird festgestellt, dass es zu beliebigem $\Phi$ immer genau einen universellen Homotopiefunktor gibt, der die Eigenschaft $W$ ) hat.

1. Die Kategorie $\mathfrak{A}$. In einer Gruppe $G$ führen wir für zwei Elemente $a, b \in G$ die folgende " $\leqq$-Relation ein: Es ist $a \leqq b$, wenn für jeden Homomorphismns $f: G^{\prime} \rightarrow G$, in dessen Bildbereich $b$ liegt, auch $a$ im Bild von $f$ liegt. Man prüft sofort nach: Es ist $a \leqq b$ genau dann, wenn für die von $a$ bezw. von $b$ erzeugten zyklischen Untergruppen gilt:

$$
\{a\} \leqq\{b\}
$$

Diese Relation ist eine schwache teilweise Ordnung, d. h. es gilt:

1 Mit Koeffizienten in $R_{1}$ (= reelle Zahlen $\bmod 1$ ). 
01) $a \leqq a$

$02) \quad a \leqq b, b \leqq c \Rightarrow a \leqq c$

aber nicht mehr notwendigerweise

0 3) $a \leqq b, b \leqq a \Leftrightarrow a=b$.

Man kann aber beim Fehlen von 03 ) sofort zu einer Klasseneinteilung: $a \sim b \Leftrightarrow a \leqq b \leqq a$ übergehen ${ }^{2}$ und bekommt dann eine teilweise geordnete Menge (also eine solche, die 01)-0 3) erfüllt) heraus. Ist $f \in \mathbb{S}\left(\mathbb{S}=\right.$ Kategorie der Gruppen mit Homomorphismen), $f: G^{\prime} \rightarrow G$ $a^{\prime} \leqq b^{\prime}$ in $G^{\prime}$, so ist $f\left(a^{\prime}\right) \leqq f\left(b^{\prime}\right)$. Ausserdem ist ein Nullelement $0 \in G$, das Einselement der Gruppe (das wir hier unabhängig davon ob $G$ abelsch ist order nicht mit dem Buchstaben 0 bezeichnen) vorhanden. Es gilt, $0 \leqq a$ für alle $a \in G$. Folgende zusäzliche Bedingung wird von einem $f \in \mathbb{S}$ noch erfüllı:

$04)$ Ist $a \leqq f\left(b^{\prime}\right)$ in $G$, so gibt es ein $a^{\prime} \in G^{\prime}, a^{\prime} \leqq b^{\prime}$ mit $f\left(a^{\prime}\right)=a$. Die Eigenschaft 04 ) hat u. a. $f(0)=0$ zurfolge. Nun kann man die Kategorie $\mathfrak{A}$ definieren:

DeFINITION 1.1.

$\mathfrak{A} 1)$ Die Objekt von $\mathfrak{U}$ sind teilweise geordnete Mengen $V(\mathrm{~d} . \mathrm{h}$. 01)-03) ist erfüllt) mit Nullelement.

$\mathfrak{X} 2)$ Die Abbildungen $\varphi \in \mathfrak{A}$ sind anordnungserhaltende eindeutige Zuordnungen, die 04 ) erfüllen.

Man überzeugt sich sofort davon, dass $\mathfrak{X}$ wirklich eine Kategorie ist. Wir beschäftigen uns mit kovarianten Funktoren $\Phi: \mathfrak{A} \rightarrow \mathfrak{U}$ wobei $\Omega$ eine beliebige Kategorie ist, in der es nur hinreichend viele Nullobjekte und Nullabbildungen gibt. $\mathrm{Zu}$ jedem $X \in \Re$ soll es ein Nullobjekt $0_{X}$ sowie eine Nullabbildung $\omega_{X}: 0_{X} \rightarrow X$ geben (d. h. es ist für bel. $\left.f \in \Re, f: X \rightarrow Y, f \omega_{X}=\omega_{Y}\right)$.

Je zwei Nullobjekte $0_{X}, 0_{Y}$ sollen in $\Re$ äquivalent sein. Von $\Phi$ fordern wir nur das "Dimensionsaxiom": $\Phi 1)$ Es ist $\Phi\left(0_{x}\right)=0$.

Ein $S \in \Re$ heisst "sphärisch" bezgl. $\Phi$, wenn es folgende Eigenschaft hat:

$S)$ Es gibt in $\Phi(S)$ ein grösstes Element.

Mit anderen Worten: Es gibt in $\Phi(S)$ ein Element $s$, sodass für alle $a \in \Phi(S)$ gilt: $a \leqq s$. Zwei $S_{1} S^{\prime}$ heissen vergleichbar, $S \approx S_{1}^{\prime}$ wenn es ein $f: S \rightarrow S^{\prime}$ mit $\Phi(f) s=s^{\prime}$ gibt. Diese Relation wird transitir und symmetrisch fortgesetzt.

Das Axiom Ф1) wird von uns einzig und allein zu dem Zweck benutzt, hinreichend viele sphärische Objekte bereit zu haben. Hat man einen Funktor $\Phi: \AA \rightarrow \Im$ vorgelegt, wobei $\mathfrak{C}$ eine beliebige Kategorie ist, so gibt es i. A. unzählige Möglichkeiten daraus einen Funktor zu machen, der in $\mathfrak{A}$ abbildet. Man kann beispielsweise zu

${ }^{2}$ Die Klasse eines $a \in G$ nennen wir auch $a$. 
einem $X$ die Unterobjekte von $\Phi(X)$ betrachten (die teilweise geordnet sind). Ist $(5$ eine Kategorie von strukturierten Mengen und entsprechenden mengentheoretischen Abbildungen, die die Struktur erhalten, so kann man zunächst versuchen das nachzumachen, was wir eben bei der Kategorie (S) taten: Ist $C \in \mathbb{C} a, b \in C$, so setzen wir $a \leqq b$, wenn für jedes $f: C^{\prime} \rightarrow C$ in dessen Bildbereich $b$ liegt gilt, auch $a \in$ Bild $f$ ist. Allerdings muss diese Relation nicht unbedingt natürlich sein, man findet also auf diesem Wege nicht immer einen Funktor in die Kategorie $\mathfrak{A}$. Es gibt einen anderen Weg, das doch noch zu retten: In [2] wurde der Begriff der grössten Operation $U$ zu einem Funktor $\Phi$ erklärt. Nehmen wir den identischen Funktor und die dazu gehörige grösste Operation, so kann man $a \leqq b$ setzen, wenn $U(a) \leqq U(b)$ ist. Diese Definition liefert (wegen der Natürlichkeit der Operation $U$ ) eine natürliche teilweise Ordnung.

2. Der Funktor $\Phi_{\pi}$. $\mathrm{Zu} \quad \Phi: \Re \rightarrow \mathfrak{U}$ geben wir einen neuen Funktor $\Phi_{\pi}: \Re \rightarrow \mathfrak{A}$ sowie eine natürliche Transformation $h: \Phi_{\pi} \rightarrow \Phi$ an, $h \in \mathfrak{U}\left(\mathrm{d}\right.$. h. $h(X): \Phi_{\pi}(X) \rightarrow \Phi(X)$ ist für alle $X \in \mathfrak{R}$ in $\mathfrak{U}$ ) sodass ein "Hurewicz-Satz" gilt:

$H) \quad$ Ist $S$ sphärisch bezgl. $\Phi$, so gibt es ein $h^{\prime}: \Phi(S) \rightarrow \Phi_{\pi}(S)$ in A für welches

$$
h h^{\prime}=\text { Identität }
$$

ist. Dieses $h^{\prime}$ ist natürlich, d.h. für ein $f: S \rightarrow S_{1}\left(S, S_{1} \in \Re_{1} S \approx S_{1}\right.$ sphärische Objekte) gilt $h^{\prime} \Phi(f)=\Phi_{\pi}(f) h^{\prime}$.

Wir nennen auch $h^{\prime} \Phi(S)=\Phi_{\pi}(S)$ und finden, dass $h$ au $\Phi^{\prime}{ }_{\pi}(S)$ ein Isomorphismus (in der Katgeorie $\mathfrak{A}$ ) ist. Es wird sich herausstellen, dass für $\Phi_{\pi}$ noch gilt:

II) Ist $a \in \Phi_{\pi}(X), X \in \Re$ so gibt es ein sphärisches $S, f: S \rightarrow X$, $f \in \Re$ sowie ein $a^{\prime} \in \Phi_{\pi}^{\prime}(S)$, sodass $\Phi_{\pi}(f) a^{\prime}=a$ ist.

In Worten: Jedes $a \in \Phi_{\pi}(X)$ "kommt von einem $a^{\prime} \in \Phi^{\prime}{ }_{\pi}(S)$ her". Die Konstruktion eines solchen $\Phi_{\pi}$ zu gegebenem $\Phi$ verläuft folgendermassen:

Man nehme zu gegebenem $X \in \Re$ Paare $(a, f)$, wobei $a \in \Phi(S), S$ ein sphärisches Objekt und $f: S \rightarrow X$ in $\Re$ ist. Wenn $\left(a^{\prime}, f^{\prime}\right),\left(a^{\prime \prime}, f^{\prime \prime}\right)$ zwei Paare (zu dem gleichen $X$ ) sind, setzen wir $\left(a^{\prime}, f^{\prime}\right) \approx\left(a^{\prime \prime}, f^{\prime \prime}\right)$ $\left(f^{\prime}: S^{\prime} \rightarrow X, f^{\prime \prime}: S^{\prime \prime} \rightarrow X\right)$, wenn es ein Paar $(a, f)$ sowie zwei Abbildungen $r^{\prime}: S \rightarrow S^{\prime}, r^{\prime \prime}: S \rightarrow S^{\prime \prime}$ gibt, sodass

$$
f^{\prime} r^{\prime}=f=f^{\prime \prime} r^{\prime \prime}
$$


und

$$
\begin{gathered}
\Phi\left(r^{\prime}\right) a=a^{\prime} \\
\Phi\left(r^{\prime \prime}\right) a=a^{\prime \prime}
\end{gathered}
$$

ist. Wir setzen diese Beziehung transitiv fort und erhalten so eine Aquivalenzrelation zwischen Paaren $(a, f)$, die wir mit dem Symbol “ " bezeichnen wollen. Die Elemente von $\Phi_{\pi}(X)$ sollen die Äquivalenzklassen von Paaren im obigen Sinne sein. Wir nennen $\overline{(a, f)}$ die Klasse des Paares $(a, f)$. Wir geben $\Phi_{\pi}(X)$ die Struktur eines Objektes aus $\mathfrak{A}$ indem wir definieren: Es ist für zwei Paare $\left(a^{\prime}, f^{\prime}\right) \leqq$ $\left(a^{\prime \prime}, f^{\prime \prime}\right)$, wenn man $f^{\prime}=f^{\prime \prime}$ und $a^{\prime} \leqq a^{\prime \prime}$ hat. Für $a, a^{\prime} \in \Phi_{\pi}(X)$ setzen wir $\alpha \leqq \alpha^{\prime}$, wenn es ein $(a, f) \in \alpha,\left(a^{\prime}, f^{\prime}\right) \in \alpha^{\prime}$ mit $(a, f) \leqq\left(a^{\prime}, f^{\prime}\right)$ gibt. Wir haben $\mathrm{zu}$ beweisen:

\subsection{Es ist $\Phi_{\pi}(X)$ ein Objekt in $\mathfrak{2}$.}

Beweis. Zunächst ist sicherlich $\alpha \leqq \alpha$, also 0 1) erfüllt. Ist $\alpha \leqq \alpha^{\prime}, \alpha^{\prime} \leqq \alpha^{\prime \prime}$ so bedeutet das die Existenz von Paaren $(a, f) \in \alpha$, $\left(a^{\prime}, f^{\prime}\right),\left(b^{\prime}, g^{\prime}\right) \in \alpha^{\prime}$ und $\left(a^{\prime \prime}, f^{\prime \prime}\right) \in \alpha^{\prime \prime}$, sodass

$$
\begin{aligned}
(a, f) & \leqq\left(a^{\prime}, f^{\prime}\right)=\left(a^{\prime}, f\right) \\
\left(b^{\prime}, g^{\prime}\right) & \leqq\left(a^{\prime \prime}, f^{\prime \prime}\right)
\end{aligned}
$$

ist. Nun ist $\left(a^{\prime}, f^{\prime}\right) \sim\left(b^{\prime}, g^{\prime}\right)$ und wir wollen zunächst annehmen, dass $\left(a^{\prime}, f^{\prime}\right) \approx\left(b^{\prime}, g^{\prime}\right)$ ist. Wir finden ein Paar $(c, 1)$ sowie Abbildungen $r^{\prime} r^{\prime \prime}$, sodass das Diagramm

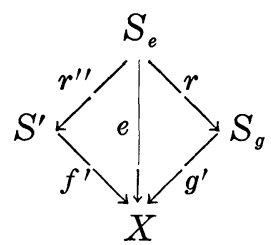

kommutativ und $\Phi\left(r^{\prime \prime}\right) c=a^{\prime}, \Phi\left(r^{\prime}\right) c=b^{\prime}$ ist. Wegen 0 4) für $\Phi\left(r^{\prime \prime}\right)$ ist ein $a_{1} \in \Phi\left(S_{1}\right)$ mit $\Phi\left(r^{\prime \prime}\right) a_{1}=a$ und $a_{1} \leqq c$ vorhanden. Es ist $\left(a_{1}, 1\right) \approx(a, f)$ (man beachte: $\left.f=f^{\prime}\right)$ und darum

$$
\alpha \leqq \alpha^{\prime \prime} .
$$

Ist nur $\left(a^{\prime}, f^{\prime}\right) \sim\left(b^{\prime}, g^{\prime}\right)$, so kann man leicht diesen Schluss iteriren und damit (3) beweisen. Damit ist 02 ) gezeigt.

Ist $\alpha \leqq \alpha^{\prime}$ und $\alpha^{\prime} \leqq \alpha$ so zeigt man mit einem ähnlichen Schluss, $\operatorname{dass} \alpha=\alpha^{\prime}$ ist.

Um den Beweis von 2.1. abzuschliessen, haben wir noch ein Nullelement $0 \in \Phi_{\pi}(X)$ mit der Eigenschaft $0 \leqq \alpha$ für alle $\alpha \in \Phi_{\pi}(X)$ 
zu finden. Man betrachte Paare $(0, f)$ wo $f: S \rightarrow X$ und 0 das Nullelement in $\Phi(S)$ ist. Wegen der Voraussetzung über $\Re$ und $\Phi$ 1) sind alle diese Paare äquivalent zueinander. Es ist sicherlich $(0, f) \leqq$ $(a, f)$ für ein beliebiges Paar $(a, f)$.

Damit ist 2.1. bewiesen.

Wir erklären nun Abbildungen $\Phi_{\pi}(g): \Phi_{\pi}(X) \rightarrow \Phi_{\pi}(Y)$ zu einem $g: X \rightarrow Y$ in $\Omega$ und weisen anschliessend nach, dass sie in $\Omega$ liegen. Ist $(a, f)$ ein Paar, $f: S \rightarrow X$, so betrachten wir das Paar $(a, g f)$ und setzen:

$$
\Phi_{\pi}(g) \overline{(a, f)}=\overline{(a, g f)} .
$$

Ist $(a, f) \approx\left(a^{\prime}, f^{\prime}\right)$, so ist trivialerweise auch $(a, g f) \approx\left(a^{\prime}, g f^{\prime}\right)$ und darum führt die so definierte Zuordnung Klassen wirklich in Klassen über. Ist $(a, f) \leqq\left(a^{\prime}, f\right)$, so ist $(a, g f) \leqq\left(a^{\prime}, g f\right)$ und damit $\Phi_{\pi}(g)$ anordnungserhaltend. Ist $\alpha \leqq \beta=\Phi_{\pi}(g) \beta^{\prime}$ in $\Phi_{\pi}(Y)$, so nehmen wir ein Paar $\left(b^{\prime}, f^{\prime}\right) \in \beta^{\prime}$ und finden nach der im Beweis von 2.1. durchgeführten Überlegung ein $(a, 1) \in \alpha$ mit $(a, 1) \leqq\left(b^{\prime}, g f^{\prime}\right)$. Das bedeutet aber: $1=g f^{\prime}$ und das Paar $\left(a, f^{\prime}\right)$ erfüllt damit die Forderung $\alpha^{\prime}=\overline{\left(a, f^{\prime}\right)} \leqq \beta^{\prime}, \Phi_{\pi}(g) \alpha^{\prime}=\alpha$. Damit ist gezeigt:

\subsection{Es ist $\Phi_{\pi}(g) \in \mathfrak{A}$.}

Die Tatsache, dass sich $\Phi_{x}(g)$ funktoriell verhält ist trivial und somit haben wir:

2.3. Es ist $\Phi_{\pi}: \Re \rightarrow \mathfrak{U}$ ein Funktor.

Die Konstruktion von $h$ ist sehr einfach:

Ist $\alpha=\overline{(a, f)} \in \Phi_{\pi}(X)$, so setzen wir:

$$
h \overline{(a, f)}=\Phi(f) a .
$$

Ist $\left(a^{\prime}, f^{\prime}\right) \approx\left(a^{\prime \prime}, f^{\prime \prime}\right)$ so weist man sofort nach, dass aus $(1),(2)$ folgt:

$$
h \overline{\left(a^{\prime}, f^{\prime}\right)}=h \overline{\left(a^{\prime \prime}, f^{\prime \prime}\right)}
$$

Das gleiche gilt demnach auch, wenn $\left(a^{\prime}, f^{\prime}\right) \sim\left(a^{\prime \prime}, f^{\prime \prime}\right)$ ist. Ist $(a, f) \leqq\left(a_{1}, f\right)$, so ist $h \overline{(a, f)} \leqq h \overline{\left(a_{1}, f\right)}$ und ebenso leicht sieht man 0 4) für $h$ ein. Also liegt $h$ in $\mathfrak{A}$. Ist $g: X \rightarrow Y$, so gilt

$$
\Phi(g) h \overline{(a, f)}=\Phi(g f) a=h \overline{(a, g f)}=h \Phi_{\pi}(g) \overline{(a, f)} .
$$

Somit ist $h$ natürlich.

2.4. Es ist $h$ eine natürliche Abbildung in $\mathfrak{A}, h: \Phi_{\pi} \rightarrow \Phi$.

Ist $\nu: S \rightarrow S$ für ein sphärisches Objekt die identische Abbildung, so 
ist folgendes richtig,

2.5. Ist $\left(a^{\prime}, \nu\right) \sim\left(a^{\prime \prime}, \nu\right)$ so ist $a=a^{\prime}$.

Beweis. Ist $\left(a^{\prime}, \nu\right) \approx\left(a^{\prime \prime}, \nu\right)$, so ist ein Paar $(a, f), f: S_{1} \rightarrow S$ vorhanden, sowie Abbildungen $r^{\prime}, r^{\prime \prime}: S_{1} \rightarrow S$ sodass gilt:

$$
\begin{gathered}
\nu r^{\prime}=\nu r^{\prime \prime}=f \\
\Phi\left(r^{\prime}\right) a=a^{\prime} \\
\Phi\left(r^{\prime \prime}\right) a=a^{\prime \prime},
\end{gathered}
$$

also ist

$$
a^{\prime}=\Phi(f) a=a^{\prime \prime} \text {. }
$$

Entsprechend behandelt man den Fall $\left(a^{\prime}, \nu\right) \sim\left(a^{\prime \prime}, \nu\right)$.

2.6. Ist $S$ ein sphärisches Objekt und $(a, f)$ ein Paar zu $S, f: S_{1} \rightarrow S$, $S \approx S_{1}$ so ist

$$
(a, f) \sim(\Phi(f) a, \nu)
$$

Beweis. Man betrachte das Diagramm:

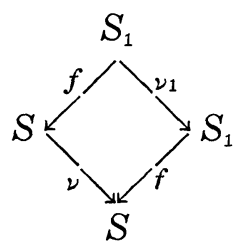

wobei $\nu_{1}$ die Identität für $S_{1}$ ist.

Trivial ist noch:

2.7. Ist $a^{\prime}, a^{\prime \prime} \in \Phi(S), a^{\prime} \leqq a^{\prime \prime}$ so ist $\left(a^{\prime}, \nu\right) \leqq\left(a^{\prime \prime}, \nu\right)$.

Aus 2.5. bis 1.7. folgt:

2.8. Erklärt man auf den sphärischen Objekten die Abbildung

$$
h^{\prime}(\alpha)=\overline{(a, \nu)},
$$

so gilt

$$
h h^{\prime}=\text { Identität }
$$

und $h^{\prime}$ ist ein natürlicher Homomorphismus in $\mathfrak{A}$, im Sinne von $H$ ) Es ist also $H$ ) für den Funktor $\Phi_{\pi}$ richtig. Ist $\alpha \in \Phi_{\pi}(X)$, so ist ein 
$\alpha^{\prime} \in h^{\prime} \Phi(S)$ für ein gewisses sphärisches Objekt $S$ vorhanden, sowie ein $f: S \rightarrow X$ mit

$$
\Phi_{\pi}(f) \alpha^{\prime}=\alpha \text {. }
$$

Man nehme einfach zu $\alpha$ ein $(a, f) \in \alpha$ und setzte $\alpha^{\prime}=\overline{(a, \nu)}=h^{\prime}(a)$. Sodann ist also auch $I$ ) erfüllt. Mit diesen Betrachtungen ist die Konstruktion eines $\Phi_{\pi} \mathrm{zu}$ gegebenem $\Phi$ abgeschlossen. Wir kommen nun zur Untersuchung der Universalitätsfrage.

3. $\Phi_{\pi}$ ist universell. Sei $\Psi$ irgend ein Funktor, $\Psi$ : $\Omega-\mathfrak{A}$ für den $H$ ) mit einem gegebenem $h_{\Psi}: \Psi-\Phi$ und einem $h^{\prime} \in \mathfrak{U}$ gilt. Wir behaupten:

3.1. Es gibt eine Abbildung $\eta: \Phi_{\pi}-T$, die mit $h, h_{\Psi_{1}} \cdots$ verträglich ist, d.h. für die die Diagramme:

(6a)

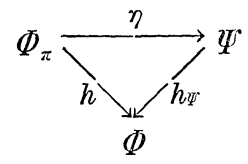

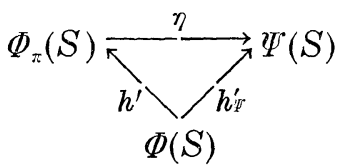

kommutativ sind ( $S$ ist ein sphärisches Objekt).

Beweis. Ist $\alpha \in \Phi_{\pi}(X),(\alpha, f) \in \alpha$, so definieren wir

$$
\eta(\alpha)=\Psi(f) h_{\psi}^{\prime}(\alpha) \text {. }
$$

Ist $\left(a^{\prime}, f^{\prime}\right) \approx\left(a^{\prime \prime}, f^{\prime \prime}\right)$, so ist (mit den Bezeichnungen von (1), (2))

$$
\begin{aligned}
\Psi\left(f^{\prime}\right) h_{y}^{\prime}\left(a^{\prime}\right) & =\Psi(f) h_{\psi}^{\prime}(a) \\
& =\Psi\left(f^{\prime \prime}\right) h_{\Psi}^{\prime}\left(a^{\prime \prime}\right) .
\end{aligned}
$$

Also ist $\eta$ von der Repräsentantenauswahl unabhängig. Ist $g: X \rightarrow Y$ in $\Re$, so ist

$$
\begin{aligned}
& \eta \Phi_{\pi}(g) \alpha=\Psi(g f) h_{\psi}^{\prime}(a) \\
& \Psi(g) \eta(\alpha)=\Psi(g) \Psi(f) h_{\psi}^{\prime}(\alpha),
\end{aligned}
$$

also ist $\eta$ natürlich. Ist $\alpha^{\prime} \leqq \alpha^{\prime \prime}$ in $\Phi_{\pi}(X)$, so nehmen wir einen Repräsentanten $\left(a^{\prime}, f\right)$ bezw. $\left(a^{\prime \prime}, f\right)$ mit $a^{\prime} \leqq a^{\prime \prime}$ und es ist dann

$$
\Psi(f) h_{\Psi}^{\prime}\left(a^{\prime}\right) \leqq \Psi(f) h_{\Psi}^{\prime}\left(a^{\prime \prime}\right),
$$

also

$$
\eta\left(\alpha^{\prime}\right) \leqq \eta\left(\alpha^{\prime \prime}\right)
$$

Ist $\xi \in \Psi(X), \xi \leqq \eta(\alpha), \alpha \in \Phi_{\pi}(X)$, so ist wegen 04$)$ für $\Psi(f) h_{\Psi}^{\prime}$ ein 
$a_{1} \leqq a \mathrm{mit}$

$$
\Psi(f) h_{\psi}^{\prime}\left(a_{1}\right)=\xi
$$

vorhanden. Also ist

$$
\eta \overline{\left(a_{1}, f\right)}=\xi
$$

und $\eta$ erfüllt 04 ) liegt also in $\mathfrak{A}$. Es gilt schliesslich:

$$
\begin{aligned}
h_{\Psi} \eta \overline{(a, f)} & =h_{\Psi} \Psi(f) h_{\Psi}^{\prime}(a)=\Phi(f) h_{\Psi} h_{\Psi}^{\prime}(a) \\
& =\Phi(f) a=h \overline{(a, f)}
\end{aligned}
$$

und

$$
\eta h^{\prime}(a)=\Psi(\nu) h_{\Psi}^{\prime}(a)=h_{y}^{\prime}(a)
$$

$a \in \Phi_{\pi}(S)$ für ein sphärisches Objekt $S$ in $\Re$. Damit ist 3.1. bewiesen. Man kann sogar zeigen:

3.2. Ist $\zeta: \Phi_{\pi} \rightarrow \Psi$ irgend eine Abbildung, die (6a), (6b) erfüllt, so ist $\zeta=\eta$.

Beweis. Sei $\alpha \in \Phi_{\pi}(X)$, so gibt es wegen $\left.\Pi\right)$ ein $a \in \Phi(S), f: S \rightarrow$ $X$ für ein gewisses sphärisches $S \in \Re$ mit $\alpha=\Phi_{\pi}(f) h^{\prime}(a)$. Also ist:

$$
\begin{aligned}
\zeta(\alpha) & =\Psi(f) \zeta h^{\prime}(a) \\
& =\Psi(f) h_{\Psi}^{\prime}(a) \\
& =\eta(\alpha) .
\end{aligned}
$$

Definition 3.1. Ein Funktor $\Omega: \Omega \rightarrow \mathfrak{A}$ heisst bezüglich einer Eigenschaft $(A)$ (die für Funktoren und Abbildungen zwischen Funktoren formuliert sein kann) universell, wenn es für jeden anderen Funktor $\Lambda: \Re \rightarrow \mathfrak{A}$ mit der Eigenschaft $(A)$ genau eine Abbildung $\phi$ mit Eigenschaft $(A), \varphi: \Omega \rightarrow \Lambda$ gibt.

\section{Der Hauptsatz}

4.1. Sind $\Phi_{\pi}$ und $\Psi$ beide universell bezgl. der Eigenschaft $H$ ) und mit den beiden Forderungen (6a), (6b) für die verbindenden Abbildungen $\eta: \Phi_{\pi} \rightarrow \Psi, \zeta: \Psi \rightarrow \Phi_{\pi} \eta, \zeta \in \mathfrak{A}$ so ist $\eta$ ein Isomorphismus.

Beweis. Es ist $\zeta \eta: \Phi_{\pi} \rightarrow \Phi_{\pi}$ eine Abbildung die (6a), (6b) erfüllt. Andererseits tut das auch die Identität, also ist $\zeta \eta=$ Identität. Aus Symmetriegründen gilt auch $\eta \zeta=$ Identität, was 4.1. beweist.

Definition 4.1. Ein Funktor $\Phi_{\pi}$, der $H$ ) bezgl eines Funktors $\Phi$ erfüllt heisst ein Homotopiefunktor zu $\Phi$. Eine natürliche Abbildung 
zwischen zwei Homotopiefunktoren $\Omega, \Lambda$ zu gleichem $\Phi$ heisst zulässig, wenn sie (6a), (6b) erfüllt.

Satz 1. Erfüllt ein Funktor $\Phi: \Re \rightarrow \mathfrak{A}$ die Bedingung $\Phi 1$ ), so gibt es bis auf Isomorphismen genau einen bezgl. zulässiger Abbildungen universellen Homotopiefunktor $\Phi_{\pi}: \Re \rightarrow \mathfrak{A} \quad z u \quad \Phi$. Es ist $\Phi_{\pi}$ ein Funktor, der die Eigenschaft $\Pi$ ) hat.

5. Hurewiczsche Homotopiegruppen. Wir gehen aus von einer Kategorie $\widetilde{\wp}$ von einfach zusammenhängenden topologischen Räumen mit Basispunkt und Homotopieklassen von stetigen (basispunkterhaltenden) Abbildungen als Morphismen. Alle Inklusionen $x_{0} \in X\left(x_{0}\right.$-Basispunkt) sollen in $\mathfrak{F}$ liegen. Auf $\mathfrak{F}$ ist der singuläre Homologiefunktor $H(*)=$ $\bigcup_{1}^{\infty} H_{n}(*)$ definiert. Wir wollen ihn ersetzen, durch einen Funktor (den wir wieder mit $H$ bezeichnen) nach $\mathfrak{A}$. Jedem $X \in \mathfrak{F}$ wird nun also die Menge der zyklischen Untergruppen $\{a\} \subseteq H_{n}(X)$ für alle $n$ zugeordnet. Ist $f \in \mathfrak{F}$ also $f$ eine Homotopieklasse von stetigen Abbildungen von $X$ nach $Y$, so ist $H(f) \in \mathfrak{A}$ erklärt. Ebenso behandeln wir den Hurewiczschen Homotopiefunktor $\pi: \Re \rightarrow \mathfrak{A}$. Setzen wir in obigen Betrachtungen $H=\Phi$, so erfüllt $\pi$ und der klassische Hurewicz-Homomorphismus $h: \pi \rightarrow H$ (der natürlich auch für unsere Funktoren, die in $\mathfrak{A}$ abbilden erklärt ist) die Forderung $H$ ). Die sphärischen Objekte sind gerade diejenigen Räume $X \in \mathfrak{F}$ (den Basispunkt lassen wir in der Schreibung fort) für die $H(X)$ eine zyklische Gruppe ist. Das aber sind zunächst Sphären $S^{n}$ und die $n+1$-Zellen, $B_{m}^{n+1}$ deren Randsphäre $S^{n}$ durch eine Abbildung vom Grade $m$ verheftet ist. Alle anderen Räume $S$, die sphärisch im Sinne von Bedingung $S$ ) sind, haben die Eigenschaft, dass es eine Abbildung $f: S^{n} \rightarrow S$ order $f: B_{m}^{n+1} \rightarrow S$ gibt, für die $H(f)$ ein Isomorphismus ist. Das ist eine wesentliche Eigenschaft der singulären Homologie, die sich mit elementaren Mitteln beweisen lässt. Wir können uns also auf Sphären $S^{n}$ und Räume $B_{m}^{n+1}$ beschränken, wenn wir sphärische Objekte untersuchen. Für diese beiden gilt aber, wie man weiss, die Aussage $H$ ).

5.1. Es erfüllt der Funktor $\pi: \mathfrak{\Im} \rightarrow \mathfrak{A}$ die Voraussetzung $H) .^{3}$ Wir zeigen, dass $\pi: \mathfrak{F} \rightarrow \mathfrak{A}$ in unserem Sinne (Definition 3.1., 4.1.) universell ist.

Sei $\Psi: \mathfrak{F} \rightarrow \mathfrak{A}$ irgend ein Funktor, der $H$ ) erfüllt, so erklären wir ein $\eta: \pi \rightarrow \Psi$ in folgender Weise: Ist $\alpha \in \pi(X), \alpha=\{a\} \cong \pi_{n}(X), a=$ $\pi(f) \nu$ ( $\nu$ ist die Homotopieklasse der Identität), so finden wir ein $\nu^{\prime} \in$ $H\left(S^{n}\right)$, sodass $h^{\prime}\left(\nu^{\prime}\right)=\nu$ ist. Wir setzen:

$$
\eta(\alpha)=\Psi(f) h_{\Psi}^{\prime}\left(\left\{\nu^{\prime}\right\}\right) \text {. }
$$

${ }^{3}$ Es ist hier $S \approx S^{\prime}$ genan dann, wenn $H_{m}(S)=\sigma \Leftrightarrow H_{m}\left(S^{\prime}\right)=\sigma$. 
Ebenso wie im 4. Abschnitt beweist man, dass $\eta$ eine zulässige Abbildung in $\mathfrak{A}$, dass es eindeutig bestimmt und damit, dass $\pi$ universell ist.

Satz 2. Ist $\mathfrak{F}$ eine Kategorie von einfach zusammenhängenden ${ }^{4}$ topologischen Räumen mit Basispunkt und basispunkterhaltenden Homotopieklassen von stetigen Abbildungen die neben dem Basispunkt $x_{0}$ auch alle Inklusionen $x_{0} \in X, X \in \widetilde{F}$ enthält, ist ferner $H: \mathfrak{F} \rightarrow \mathfrak{A}$ der singuläre Homologiefunktor so ist $H_{\pi}=\pi: \mathfrak{\mho} \rightarrow \mathfrak{A}$ der Hurewiczsche Homotopiefunktor.

6. Dualisierung. Geht man nicht, wie wir es bisher taten von einem kovarianten, sondern von einem kontravarianten Funktor $\Phi: \Re \rightarrow \mathfrak{U}$ aus, dann lassen sich alle unsere Betrachtungen wörtlich dualisieren. Man kann die Bedingung $H$ ) wörtlich übernehmen. Bei der Formulierung der dualen Bedingung $\widetilde{\Pi}$ ) zu $\Pi$ ) hat man einfach die Richtung der Abbildung $f$ (jetzt: $f: X \rightarrow S, f \in \Re$ ) umzukehren.

Wir hatten von der Kategorie $\Omega$ die Existenz von Nullobjekten und von Abbildungen $\omega_{X}: 0_{X} \rightarrow X$ gefortert, sodass für ein $f: X \rightarrow Y$ $f \omega_{X}=\omega_{Y}$ ist. Jetzt fordern wir die Existenz von Abbildungen $\zeta_{X}: X \rightarrow 0_{X}$, sodass für ein $f: Z \rightarrow X \zeta_{X} f=\zeta_{Z}$ ist. Die Bedingung $\Phi$ 1) bleibt wörtlich erhalten. Der Funktor $\Phi_{\pi}$, den wir nunmehr konstruieren ist jetzt natürlich kontravariant und wir haben:

Satz 1. Erfüllt ein kontravarianter Funktor $\Phi$ die Bedingung $\Phi 1)$, so gibt es bis auf Isomorphismen genau einen bezgl. zulässiger Abbildungen universellen kontravarianten Homotopiefunktor $\Phi_{\pi}: \Re \rightarrow \mathfrak{A} z u \Phi$.

7. Cechsche Kohomologiegruppen. Wir gehen aus von einer Kategorie $\mathfrak{F}$ von topologischen Räumen mit Basispunkt und Homotopieklassen von stetigen Abbildungen als Morphismen. Alle Basispunkte $x_{0} \in X \in \mathfrak{F}$ und alle Abbildungen $X \rightarrow x_{0}$ sollen in $\mathfrak{F}$ enthalten sein. Ferner sollen alle Räume in $\mathfrak{F}$ den Homotopietyp eines CW-Komplexes haben und einfach zusammenhängend sein. Wir konstruieren nun einen Funktor $\tilde{\pi}: \mathfrak{F} \rightarrow \mathfrak{A}$ auf folgende Weise: Es ist

$$
\tilde{\pi}(*)=\bigcup \operatorname{Uom}\left(\pi_{n}(*), R_{1}\right) \quad(* \in \mathfrak{F})
$$

wo $R_{1}$ die Gruppe der reellen Zahlen mod 1 ist, ein Funktor nach \&. So wie im ersten Abschnitt machen wir daraus einen Funktor nach

${ }^{4}$ Man könnte auch ohne den einfachen Zusammenhang für ein $X \in \mathfrak{F}$ auskommen, wenn man von den sphärischen Objekten $S \in \mathfrak{F}$ voraussetzt, dass sie einfach zusammenhängend sind. 
I. Für zwei $\varphi_{i}: \pi_{n_{i}}(X) \rightarrow R_{1}$ setzen wir demnach $\varphi_{1} \geqq \varphi_{2}$ wenn $n_{1}=$ $n_{2}=n$ ist und wenn es ein $r: R_{1} \rightarrow R_{1}$ in der Form $r(a)=m a$ (für festes ganzzahliges $m$ ) mit $r \varphi_{1}=\varphi_{2}$ gibt. Die Klasse eines $\varphi: \pi_{n}(X) \rightarrow R_{1}$ bezeichnen wir mit $\bar{\varphi}\left(\varphi_{1} \sim \varphi_{2} \Leftrightarrow \varphi_{1} \leqq \varphi_{2} \leqq \varphi_{1}\right)$. Auf diese Weise haben wir einen Funktor nach $\mathfrak{N}$, den dualen Homotopiefunktor $\tilde{\pi}$. Wir konstruieren nun $\tilde{\pi}_{\pi}$ und wol en beweisen, dass $\tilde{\pi}_{\pi}=$ $H^{*}$, der von der Cechschen Kohomologie (mit Koeffizienten in $R_{1}$ ) induzierte Funktor nach $\mathfrak{A}$ ist: $H^{*}(X)=\bigcup H^{n}\left(X, R_{1}\right)=\mathrm{U}\left[X, K\left(R_{1}, n\right)\right]$. Wie üblich unterscheiden wir in der Bezeichnung nicht den Funktor nach (S) und den zugeordneten nach $\mathfrak{A}$. Zunächst interessieren wir uns für die sphärischen Objekte von $\tilde{\pi}$.

7.1. Jedes bezgl. $\tilde{\pi}$ sphärische Objekt $T \in \mathfrak{F}$ ist ein $K(G, n)$ für eine gewisse Gruppe $G(n>1)$.

Beweis. Ist $T$ sphärisch bezgl. $\tilde{\pi}$, so sieht man, dass es nur eine Dimension $n$ mit $\pi_{n}(T) \neq 0$ geben kann, weil es sonst kein maximales $\varphi: \pi_{n}(T) \rightarrow R_{1}$ gibt. Zwei nichttriviale $\varphi_{i}: \pi_{n_{i}}(X) \rightarrow R_{1}$ sind unvergleichbar, wenn $n_{1} \neq n_{2}$ ist. Ist aber ein $\pi_{n}(T) \neq 0$, so gibt es immer ein $\varphi: \pi_{n}(T) \rightarrow R_{1}, \varphi \neq 0$. Das bedeutet, dass $\mathrm{T}$ ein $K(G, n)$ ist.

Wir betrachten nun den Funktor $H^{*}$ als Funktor nach $\mathfrak{A}$ und behaupten, dass für ihn die Bedingung $H$ ) erfüllt ist. Zunächst haben wir den dualen Hurewicz-Homomorphismus anzugeben, $\widetilde{h}: H^{*} \rightarrow \tilde{\pi}$ (in भ). Sei $\bar{a} \in H^{*}(X), a \in H^{n}(X)$, so gibt es ein $f: X \rightarrow K\left(R_{1}, n\right)$ mit $f=a$. (es ist ein $f \in \mathfrak{F}$ bereits eine Homotopieklasse einer stetigen Abbildung) Wir setzen: $\widetilde{h}(a)=\pi_{n}(f)\left(: \pi_{n}(X) \rightarrow R_{1}\right)$ und erkennen, dass wir so Klassen $\bar{a}$ aus $H^{*}(X) \in \mathfrak{A}$ in Klassen $\bar{\varphi}$ aus $\tilde{\pi}(X) \in \mathfrak{A}$ überführen. Man prüft leicht nach, dass $\tilde{h}$ eine natürliche Abbildung aus $\mathfrak{A}$ ist. Sei $T$ ein sphärisches Objekt bezgl. $\tilde{\pi}$ also nach 7.1. ein $K(G, n)$. Ist $\varphi: \pi_{n}(K(G, n)) \rightarrow \pi_{n}\left(K\left(R_{1}, n\right)\right)$ so kann man $\varphi$ durch eine Abbildung $f: K(G, n) \rightarrow K\left(R_{1}, n\right)$ realisieren (nach bekannten Sätzen gibt es sogar ein eindeutig bestimmtes $f \in \widetilde{\mho}$ mit $\left.\pi_{n}(f)=\varphi\right)$ und es ist:

$$
\tilde{h}(f)=\bar{\varphi} \text {. }
$$

Dieses Argument liefert den Beweis, dass es ein natürliches $\widetilde{h}^{\prime}: H^{*}(T) \rightarrow \tilde{\pi}(T)$ gibt, sodass $\widetilde{h} \widetilde{h}^{\prime}=$ Identität ist. Die Einzeheiten des Beweises seien dem leser überlassen.

7.2. Es genügt $H^{*}$ der Bedingung $H$ ).

Der Beweis dafür, dass $H^{*}$ universell ist, ist eine unmittelbare Dualisierung des entsprechenden Beweises für $\pi$ im 5. Abschnitt. Zusammenfassend können wir formulieren: 
Satz 3. Sei $\mathfrak{F}$ eine Kategorie von topologischen Räumen mit Basispunkt wie in Satz 2, deren Objekte alle den Homotopietyp eines einfach zusammenhängenden $C W$-Komplexes haben und in der alle Projektionen $X \rightarrow x_{0}$ enthalten sind $\left(x_{0} \in X \in \widetilde{F}\right), \tilde{\pi}=\mathrm{U} \operatorname{Hom}\left(\pi_{n}, R_{1}\right)$ : $\mathfrak{F} \rightarrow \mathfrak{A}$ der duale Homotopiefunktor, so ist

$$
\tilde{\pi}_{\pi}=H^{*},
$$

der Cechsche Kohomologiefunktor (mit Koeffizienten in $\left.R_{1}\right): H^{*}(X)=$ $\mathrm{U}\left[X, K\left(R_{1}, n\right)\right]$.

8. Der Whitehead-Satz. Ist $\Phi: \Re \rightarrow \mathfrak{A}$ ein Funktor der $\Phi 1)$ erfüllt, so sagen wir, es gelte für einen anderen Funktor $\Psi: \Re \rightarrow \mathfrak{A}$ (etwa beide kovariant) eine Whitehead-Bedingung (bezgl. $\Phi$ ), wenn folgendes erfüllt ist:

$W)$ Ist $f: X \rightarrow Y$ in $\Re$ und $\Phi(f)$ ein Isomorphismus, so auch $\Psi(f)$.

In diesem Abschnitt wollen wir nur solche Homotopiefunktoren betrachten, die einer Whitehead-Bedingung bezgl. des vorgelegten $\Phi$ genügen. Wir können dann beweisen, dass es genau einen bezgl. dieser Eigenschaft und zulässiger Abbildungen universellen Homotopiefunktor gibt.

Zum Beweis dieser Tatsache betrachten wir zu dem Paar $\Re, \Phi$ eine neue Kategorie $\Omega^{\Phi}$ die wir folgendermassen konstruieren: Die Objekte von $\AA^{\Phi}$ sind die gleichen wie die Objekte von $\Omega$. Um zu einer Abbildung $F: X \rightarrow Y$ in $\AA^{\Phi}$ zu gelangen, betrachten wir eine Kette

$$
X \stackrel{f_{1}}{\longrightarrow} X_{1} \stackrel{f_{2}}{\longleftarrow} X_{2} \stackrel{f_{3}}{\longrightarrow} \cdots \stackrel{f_{n-1}}{\longleftarrow} X_{n-1} \stackrel{f_{n}}{\longrightarrow} X_{n}=Y
$$

von Abbildungen in $\Re$, wobei alle $f_{2 n}$ die Eigenschaft haben, dass $\Phi\left(f_{: n}\right)$ ein Isomorphismus ist. Die Klasses der Abbildungen $g$ mit $\Phi(g)=$ Isomorphismus nennen wir $I(\Phi)$. Diese Ketten selbst sind aber nicht die Abbildungen in $\Omega^{\Phi}$ sondern wir müssen für eine Kette erst noch einen Reduktionsprozess erklären, der aus einer Folge von Schritten der folgenden Art besteht:

(*) Man betrachte zwei aufeinanderfolgende Abbildungen in der Kette (die wir einfach $a$ und $b$ nennen wollen) und ersetze sie, wenn möglich durch eine Abbildung $c \in \Re$, so wie es in einem der kommutativen Diagramme
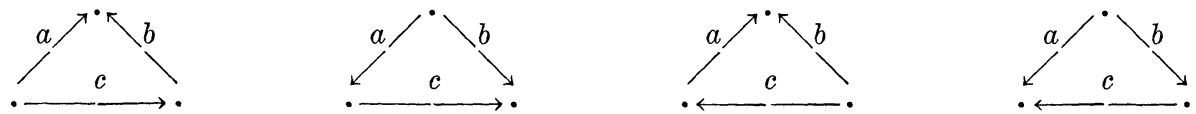
angezeigt ist. Im dritten und vierten Falle muss $c \in I(\Phi)$ sein. Nun fasse man die Abbildung $c$ im ersten und vierten Falle mit der auf $b$ folgenden, im zweiten und dritten Falle mit der $a$ vorangehenden Abbildung (falls es in der Kette eine solche Abbildung gibt) zusammen und man erhält wieder eine Kette $k^{\prime}$. Dabei beachte man, dass wenn zwei aufeinanderfolgende Abbildungen beide zu $I(\Phi)$ gehören, das Kompositum zu $I(\Phi)$ gehört. Die Länge von $k$ (die Zahl $n$ ) hat sich bei jedem Reduktionsschritt vermindert. Der Anfang und das Ende von $k$ (nämlich die Objekte $X$ und $Y$ ) sind fest geblieben.

Zwei Ketten $k_{1}, k_{2}$ heissen nun äquivalent, wenn sie gleichen Anfang und gleiches Ende haben und wenn man eine Folge von endlich vielen Reduktionen der Form (*) finden kann, die eine in die andere $\left(k_{1}\right.$ in $k_{2}$ oder $k_{2}$ in $\left.k_{1}\right)$ überführt. Diese Beziehung setzen wir transitiv fort und machen damit aus ihr eine Äquivalenzrelation. Die Abbildungen in $\Omega^{\Phi}$ sind nun Klassen $F=\bar{k}$ von Ketten $k$. Sicherlich ist $\AA^{\Phi}$ eine Kategorie: Man setzt zwei Ketten $k_{1}: X \rightarrow Y$, $k_{2}: Y \rightarrow Z$ zu einer Kette $k_{2} k_{1}$ zusammen, indem man sie hintereinander schreibt und, wenn man will bei $Y$ noch die Identität einschaltet, damit (formal) die Kettenbedingung erfüllt ist. Damit ist auch eine Komposition von Kettenklassen definiert und $\Re^{\infty}$ erfüllt die Forderungen an eine Kategorie. Ist $g: X \rightarrow Y$ in $\Re$, so ist $g$ eine spezielle (und nicht mehr reduzierbare) Kette. Ist $f: X \rightarrow Y, g: Y \rightarrow Z$ in $\Re$ so ist die Klasse $\overline{g f}$ in $\AA^{\Phi}$ mit der Klasse $\overline{g f}$ identisch. Ist $\nu: X \rightarrow X$ die Identität, so ist $\bar{\nu}$ die Identität in $\Re^{\Phi}$. Also gibt es einen Funktor $M: \Re \rightarrow \Re \Phi$ der die Objekte in sich und die Abbildungen in ihre Klassen überführt. Ist $\Psi: \Re \rightarrow \mathfrak{A}$ ein Funktor, für den jedes $g \in I(\Phi)$ einen Isomorphismus induziert, so kann man einen (sogar eindeutig bestimmen) Funktor $\Psi^{\prime}: \Re \rightarrow \mathfrak{A}$ finden, der $\Psi$ fortsetzt. Ist umgekehrt $\Psi^{\prime}: \mathfrak{R}^{\Phi} \rightarrow \mathfrak{U}$ ein beliebiger Funktor so nehme man den Funktor $\Psi^{\prime} M: \Re \rightarrow \mathfrak{A}$ und hat einen Funktor $\Psi: \Re \rightarrow \mathfrak{A}$ für den $W$ ) gilt.

8.1. a) Es gibt einen Funktor $M: \Re \rightarrow \Re^{\Phi}$ der auf den Objekten die Identität ist.

c) Erfüllt $\Psi: \Re \rightarrow \mathfrak{A}$ die bedingung $W$ ) bezgl. $\Phi$, so gibt es genau eine Fortsetzung $\Psi^{\prime}: \AA^{\Phi} \rightarrow \mathfrak{A}$.

b) Ist $\Psi^{\prime}: \mathfrak{R}^{\Phi} \rightarrow \mathfrak{U}$ so erfüllt $\Psi=\Psi^{\prime} M: \Re \rightarrow \mathfrak{A}$ die Bedingung $W$ ).

Nur c) bedarf eines Beweises: Sei $g \in I(\Phi), g: X \rightarrow Y$, so ist $g$ eine Äquivalenz in $\AA^{\Phi}$, denn es gibt in $\Omega^{\Phi}$ eine Umkehrung zu $\bar{g}$ (nämlich wieder $\bar{g}$, wobei jetzt aber $g$ als Kette mit nur einem Glied verstanden wird, wobei man, um die formale Kettenbedingung $\mathrm{zu}$ erfüllen noch die jeweilige Identität davor und dahinter schaltet). Aus funktoriellen Gründen ist $\Psi^{\prime}(\bar{g})=\Psi(g)$ ein Isomorphismus in $\mathfrak{A}$.

Wir wenden nun Satz 1 auf $\Phi^{\prime}: \mathfrak{\Re}^{\oplus} \rightarrow \mathfrak{A}$ an und erhalten ein 
$\Phi_{\pi}^{\prime}: \Re^{\Phi} \rightarrow \mathfrak{A}$. Wir setzen

$$
\Phi_{W}=\Phi_{\pi}^{\prime} M: \Re \rightarrow \mathfrak{A}
$$

Ist $\Psi: \Re \rightarrow \mathfrak{A}$ irgend ein Funktor, der $H$ ) und $W$ ) bezgl. $\Phi$ erfüllt, so kann man $\Psi^{\prime}: \mathfrak{R}^{\oplus} \rightarrow \mathfrak{A}$ bilden und auch $\Psi^{\prime}$ erfüllt $H$ ). Es gibt also eine eindeutig bestimmte zulässige Abbildung $\eta^{\prime}: \Phi_{\pi}^{\prime} \rightarrow \Psi^{\prime}$. Durch $\eta=\eta^{\prime} M: \Phi_{W} \rightarrow \Psi$ ist eine zulässige Abbildung die ebenfalls, durch $\Phi_{W}$ und $\Psi$ eindeutig bestimmt ist (da $\Re$ und $\Re^{\Phi}$ die gleichen Objekte haben) definiert und $\Phi_{W}$ ist ein bezgl. $W$ ) und zulässiger Abbildungen universeller Funktor.

Satz 4. Zu jedem Funktor $\Phi: \Re \rightarrow \mathfrak{A}$ der $\Phi$ 1) erfüllt gibt es bis auf Isomorphismen genau einen Homotopiefunktor $\Phi_{w}: \Re \rightarrow \mathfrak{A}$ für den $W$ ) gilt und der universell (mit zulässigen Abbildungen in $\mathfrak{A}$ ) bezgl. dieser Eigenschaft ist.

Satz 5. Ist $\widetilde{\jmath}$ eine Katgeorie von topologischen Räumen wie in Satz 2, deren Objekte alle einfach zusammenhängend sind, so ist

$$
H_{W}=\pi \text {. }
$$

Beweis. Es erfüllt $\pi$ die Forderung $W$ ) und es ist $\pi$ nach Satz 2 sogar ohne ausdrückliche Voraussetzung von $W$ ) universell bezgl. $H$ ). Also ist $\pi$ erst recht universell bezgl. $H$ ) und $W$ ).

\section{LITERATUR}

1. F. W. Bauer, Homotopie und Homologie, Mathem. Ann. 149 (1963), S. 105-130.

2. —_, Operationen, Crelles Journal Bd. 220, 3/4 (1965), 186-214.

Received December 6, 1965. 


\section{PACIFIC JOURNAL OF MATHEMATICS}

\section{EDITORS}

H. SAMELSON

Stanford University

Stanford, California

J. P. JANS

University of Washington

Seattle, Washington 98105
J. DugundJI

University of Southern California

Los Angeles, California 90007

RICHARD ARENS

University of California

Los Angeles, California 90024

\section{ASSOCIATE EDITORS}

E. F. BECKENBACH

B. H. NeumanN

F WolF

K. YOSIDA

\section{SUPPORTING INSTITUTIONS}

UNIVERSITY OF BRITISH COLUMBIA

CALIFORNIA INSTITUTE OF TECHNOLOGY

UNIVERSITY OF CALIFORNIA

MONTANA STATE UNIVERSITY

UNIVERSITY OF NEVADA

NEW MEXICO STATE UNIVERSITY

OREGON STATE UNIVERSITY

UNIVERSITY OF OREGON

OSAKA UNIVERSITY

UNIVERSITY OF SOUTHERN CALIFORNIA
STANFORD UNIVERSITY

UNIVERSITY OF TOKYO

UNIVERSITY OF UTAH

WASHINGTON STATE UNIVERSITY

UNIVERSITY OF WASHINGTON

AMERICAN MATHEMATICAL SOCIETY CHEVRON RESEARCH CORPORATION TRW SYSTEMS

NAVAL ORDNANCE TEST STATION

Printed in Japan by International Academic Printing Co., Ltd., Tokyo Japan 


\section{Pacific Journal of Mathematics}

\section{Vol. 21, No. $1 \quad$ November, 1967}

Friedrich-Wilhelm Bauer, Der Hurewicz-Satz................... 1

D. W. Dubois, A note on David Harrison's theory of preprimes . ......... 15

Bert E. Fristedt, Sample function behavior of increasing processes with stationary, independent increments ..................... 21

Minoru Hasegawa, On the convergence of resolvents of operators....... 35

Søren Glud Johansen, The descriptive approach to the derivative of a set function with respect to a $\sigma$-lattice ....................... 49

John Frank Charles Kingman, Completely random measures ............ 59

Tilla Weinstein, Surfaces harmonically immersed in $E^{3} \ldots \ldots \ldots \ldots \ldots . . \ldots 9$

Hikosaburo Komatsu, Fractional powers of operators. II. Interpolation spaces ......................................... 89

Edward Milton Landesman, Hilbert-space methods in elliptic partial differential equations ...................................... 113

O. Carruth McGehee, Certain isomorphisms between quotients of a group algebra ........................................ 133

DeWayne Stanley Nymann, Dedekind groups .................. 153

Sidney Charles Port, Hitting times for transient stable processes ......... 161

Ralph Tyrrell Rockafellar, Duality and stability in extremum problems involving convex functions . ............................ 167

Philip C. Tonne, Power-series and Hausdorff matrices . . .............. 189 